\title{
Function Point Metrics Improvement and Application in E-Commerce
}

\author{
Siping $\mathrm{He}$ \\ School of Information, Renmin University of China, Beijing 100872, P.R. China \\ hesiping@hotmail.com
}

\begin{abstract}
Function point measurement is one of the approaches in measuring functionality of software products, but it has many limitations. In this paper, we improve the function point measurement. The improvement mainly includes adding new factors and adjusting the calculation process. This improvement is applied in one of E-commerce project and gets appropriate result which can guide the process of software development.
\end{abstract}

Keywords: Electronic commerce, J2EE, Quality control, Testing, Function point, Measurement

\section{INTRODUCTION}

E-Commerce develops quickly in recent years, and promotes the usage of new technology, such as new frame, design pattern and program language. Tradition software development is based on integrate application software, which is composed by various modules, developing for special application, environment or organization. New software development is based on components which provide multi-application and different structure environments and organizations. Software development which based on components emphasizes interface and regulation, i.e. function. Implement is encapsulation of components, so that the requirement of clients can be realized independently. This face-on-function pattern which emphasizes on realization independently inosculates with function point analysis naturally.

Above-mentioned usage of new technology in E-commerce requires new concepts and methods in software metrics. The strength comes from key requirements in Ecommerce: popularization of Web operation model and extend application, time shortage of coming into the market, shortage of skillful developers, rapid evolution of technology, generally recognize of component-based regulation, globalization tendency and the last but not the least-expectation of the client. The strength of this transition of the pattern is the mainstream of the software development, which is Java and related enterprise regulation: Enterprise JavaBean (EJB) and Java2 Enterprise Environment (J2EE). Although other standard component is still here, EJB and standard of J2EE have become fact standard in E-Commerce.

In software metrics, the amount of functionality inherent in a product paints a picture of product size. As a distinct attribute, functionality captures an intuitive 
notion of the amount of function contained in a delivered product or in a description of how the product is supposed to be. Function Point (FP) measurement is an approach brought forward by Albrecht [1] to measure the functionality of software products. In this paper, the approach of function point measurement will be improved under new technology framework and will be used in a new E-commerce mode.

\section{INTRODUCTION OF E-COMMERCE AND FRAMEWORK}

E-commerce is the abbreviation for electronic commerce. A way of doing real-time business transactions via telecommunications networks, when the customer and the merchant are in different geographical places. Electronic commerce is a broad concept that includes virtual browsing of goods on sale, selection of goods to buy, and payment methods. Electronic commerce operates on a bona fide basis, without prior arrangements between customers and merchants. E-commerce operates via the Internet using all or any combination of technologies designed to exchange data (such as EDI or e-mail), to access data (such as shared databases or electronic bulletin boards), and to capture data (through the use of bar coding and magnetic or optical character readers) [2].

New information technology is widely used in E-commerce, such as new frameworks: Struts, Spring and Hibernate. These are all new frameworks in J2EE development. Framework is composed by a lot of classes, which provide a reusable design for application programs or we called it-a layer in a software. The code in the application call the class library to carry out the task and the framework take charge of calling the application code to manage the flow of the program. This is a so-called Hollywood principle that 'Don't call us, we will call you.' In the runtime, developer's code will be called by the framework.

\section{IMPROVEMENT IN FUNCTION POINT METRICS}

Function points are intended to measure the amount of functionality in a system as described by a specification. Function points can be computed without forcing the specification to conform to the prescripts of a particular specification model or technique. In tradition approach, to compute the number of function points, FP, we first compute an unadjusted function point count, UFC. To do this, we determine from some representation of the software the number of items of the following types: external inputs, external outputs, external inquiries, external files and internal files. After this, we calculate an adjusted function point count, FP, by multiplying UFC by a technical complexity factor.

Function points are also used in other ways as a size measure. For example, we can express defect density in terms of defects per function point. They are also used in contracts, both to report progress and to define payment. For instance, Onvlee claims that $50-60 \%$ of software contracts in the Netherlands have their costs tied to a function-points specification [3]. In other words, many companies write software 
contracts to include a price per function point, and others track project completion by reporting the number of function points specified, designed, coded and tested.

Although function points are proposed as a technology-independent measure of size, there are several problems with the function-points measure, and users of the technique should be aware of its limitations especially when technology has developed so quickly. Comparing with traditional Client/Server model, new Ecommerce software normally based on Web whose development has many new characters. The GUI (Graphical User Interface) in Web application is different which affect the computing of external input, external output and external inquiries. Web GUI generally use different components which need complex dynamic queries, so the proportion between external inquiries and external input/external output is higher than traditional Client/Server model.

In order to overcome above-mentioned limitations, we should adjust the method in computing function points. First we design a function point estimate table and organize a team of experts to fill in the table. Those experts include project manager, senior structure designer, senior function point expert. After finishing the table, project manager will discuss it with customers and affirm that every function point in the table is exact and self-contained. In this table, we add new factors: subjective risk factor and reuse factor. Subjective risk factor is divided into three grades: low, middle, high, and reuse factor has two sides: reuse and no-reuse. In our improvement, we will consider above new factors.

\section{APPLICATION IN E-COMMERCE}

In a new E-commerce model-BAB [4], the improvement of function point measurement is used and estimate the workload in the project. First of all, let's see the meaning of the new model $\mathrm{BAB}$. As we known, E-commerce can be divided into three traditional models: $\mathrm{B}$ to $\mathrm{B}, \mathrm{B}$ to $\mathrm{C}$ and $\mathrm{B}$ to $\mathrm{G}$. Here $\mathrm{B}$ means Business; $\mathrm{C}$ means Client and $G$ means Government. All of these three models do not pay attention to the middle node. But in this new E-commerce model, the middle node is emphasized and called Agent. Agent is a economics term, an entity which has independent status, and should not be considered as person or software narrowly.

For the whole E-commerce model project, we use the improvement method to evaluate the function points and get results as follow. Take one function, show the order form, as example, external inputs are 6, external outputs are 0 , external inquiries are 0 , external files are 0 , and internal files are 25 , the sum is 31 . Other factors include function points complexity weight is average, reuse factor is $\mathrm{R}$-reuse, and subjective risk factor is $\mathrm{M}$-middle. Therefore based on average productivity, 33.3 function points per month per person, we can estimate that it will take 20 days person to finish this function point. For the whole project, there are 302 function points and will take 276 days person. 


\section{CONCLUSION AND FUTURE WORK}

In this paper we improve the measure of the function points and apply it on an Ecommerce project. The improvement mainly adds two new factors and adjusts the calculation process. Comparing with tradition method, the new method considers more factors and the advancement of the technique. The result is more exactly and can be used in many aspects such as project control. In the middle of the project, FPE table can be used to master the plan of the whole project and it will be adjusted after discussion to decide the size and remain time of the project. The adjustment in function points measurement can still be improved in many aspects such as considering the components and adjusting the weights in the calculation.

\section{REFERENCES}

1. A.J. Albrecht, Measuring application development, in Proc. of IBM Applications Development Joint SHARE/GUIDE Symposium (Monterey, CA, 1979), pp. 83-92.

2. M. Fang, Electronic Commerce (Tsinghua University Press: Beijing, Beijing, 2002), pp. 4-5.

3. J. Onvlee, Use of function points for estimation and contracts, in Software Quality Assurance and Measurement, eds. N. Fenton, R.W. Whitty, and Y. Iizuka (International Thomson Computer Press: Boston, MA, 1995), pp. 88-93.

4. Y. Chen and M. Fang, Research and Practice in BAB Based on Resource Management, Renming University of China (2006). http://www.cnbab.com/bab/bab.jsp (Accessed March 4, 2007). 